\title{
Surgical treatment for acute spinal cord injury study pilot study \#2: evaluation of protocol for decompressive surgery within 8 hours of injury
}

\author{
Wai Pui Ng, M.D., Michael G. Fehlings, M.D., Ph.D., Brian Cuddy, M.D., Curtis Dickman, M.D., \\ Mahmood Fazl, M.D., Barth Green, M.D., Patrick Hitchon, M.D., Bruce Northrup, M.D., Volker \\ Sonntag, M.D., Frank Wagner, M.D. and Charles H. Tator M.D. Ph.D. \\ Division of Neurosurgery, The University of Toronto, The Toronto Hospital, Canada; and Members of \\ the STASCIS Surgical Decompression Study Group
}

Acute spinal cord injury (SCI) is a major public health problem for which there is still only limited treatment available. The National Acute Spinal Cord Injury Study-2 (NASCIS-2) and -3 clinical trials demonstrated that the use of acute pharmacotherapy with methylprednisolone can attenuate the secondary injury cascade if administered within 8 hours of acute SCI. However, no trial has been performed to examine whether acute surgical decompressive procedures within this critical 8-hour time window can improve patients' neurological outcome. The purpose of the current prospective Surgical Treatment for Acute Spinal Cord Injury Study (STASCIS) pilot study was to determine the feasibility of obtaining a radiological diagnosis of spinal canal compromise of $25 \%$ or more and to perform spinal cord (C3-T1) decompressive procedures by 8 hours postinjury. One of the following three decompressive methods was used: 1) traction alone; 2) traction and surgery; or 3) surgery alone. Twenty-six patients from eight North American centers were entered into the study between 1996 and 1997. Significant difficulties were encountered in many centers in performing immediate magnetic resonance imaging examination in patients with acute SCI. Fewer than $10 \%$ of acute cervical SCI patients could be enrolled into this protocol mainly because the combination of the required time for rescue, resuscitation, transport, imaging study, and surgical preparation exceeded the 8-hour injury-to-decompressive surgery window. Eleven patients underwent decompressive procedures initially by being placed in traction at a mean time of 10.9 hours postinjury. Those patients not undergoing this procedure underwent decompressive surgery at a mean time of 40.1 hours. However, the surgical decompressive procedure was completed within 12 hours in seven patients. As a result of these findings, several major changes have been made to the STASCIS protocol for early decompressive therapy.

Key Words * acute spinal cord injury * cervical spinal cord * magnetic resonance imaging * traction

Acute spinal cord injury (SCI) is a major public health problem for which there is still only limited 
treatment available. There are approximately 11,000 cases of acute SCI per year in North America; of the majority of these cases involve the cervical spinal cord.[15] The estimated annual cost to support and treat all patients with a SCI is over $\$ 4$ billion.[20] Accordingly, the development of improved treatment modalities would be of enormous clinical and economic benefit. At present, surgical treatment is commonly performed in patients with acute SCI and involves decompressive surgery in the spinal cord and stabilization of the unstable spine following management of the patient's cardiopulmonary and general medical status.[14,16,21] Traction is also frequently applied as a decompressive method, either alone or followed by surgery.[1,8] There is both experimental and clinical evidence to suggest that performing early decompressive procedures to treat the injured cord may result in improved neurological recovery.[1,7-9,13] The recent National Acute Spinal Cord Injury Study (NASCIS)-2 clinical trial provided evidence that the use of acute pharmacotherapy with methylprednisolone can attenuate the secondary injury cascade if administered within 8 hours of acute SCI.[5] To date, however, no trial has been performed to examine whether acute decompressive procedure undertaken within this critical 8 -hour window can improve patients' clinical and neurological outcome.

Determination of the value of acute decompressive therapies is the most important clinical issue to be addressed in the management of acute SCI.[16,23] This Surgical Treatment for Acute Spinal Cord Injury Study (STASCIS) pilot study has been designed as a preparatory step toward determining whether performing acute decompressive surgery in the cervical spinal cord (C3-T1) within 8 hours of acute SCI can improve patients' clinical and neurological outcome as compared with the more standard treatment in which decompressive procedures are performed after 24 hours or more of medical management. If the STASCIS pilot study shows that the protocol is feasible, a randomized controlled trial of acute decompression within 8 hours of injury versus subacute decompressive surgery after 24 hours or longer of medical management would be instituted.

\section{CLINICAL MATERIAL AND METHODS}

The main purposes of the STASCIS pilot study were to assess the feasibility and safety of performing acute decompressive procedures within 8 hours postinjury. Can the surgeons at the eight participating North American centers (see Appendix) perform surgical decompressive procedures in the cervical spinal cord (C3-T1) within 8 hours of trauma in cases of complete and incomplete spinal cord injury? Can early decompressive therapy be performed with an acceptable complication rate? Can decompressive therapy be accomplished in a high proportion of patients? To address these questions, each of the participating centers was asked to perform the acute decompressive therapy within 8 hours in as many patients as possible between October 1, 1996, and January 31, 1997.

The protocol, summarized in Fig. 1, required the use of immediate neuroimaging by magnetic resonance (MR) imaging or computerized tomographic (CT)/myelography (before any traction could be applied for decompression) followed by acute decompressive surgery by 8 hours postinjury in those patients in whom imaging studies revealed spinal cord compression. One of the following three decompressive methods was to be used: 1) traction alone; 2) traction and surgery; or 3) surgery alone. Patients were not randomized, and specific consent for the study was not deemed necessary. Because surgical technique varied according to the location of injury in the spine and the nature of the injury, surgeons were not restricted in their surgical technique. Imaging studies were repeated as soon as possible after the decompressive procedure but within a maximum of 7 days posttreatment to assess the adequacy of the treatment. Outcome was assessed at 6 weeks and 6 months by using the American Spinal Injury Association ASIA[3] and NASCIS[5,6] systems to score neurological status and motor and sensory 
recovery.

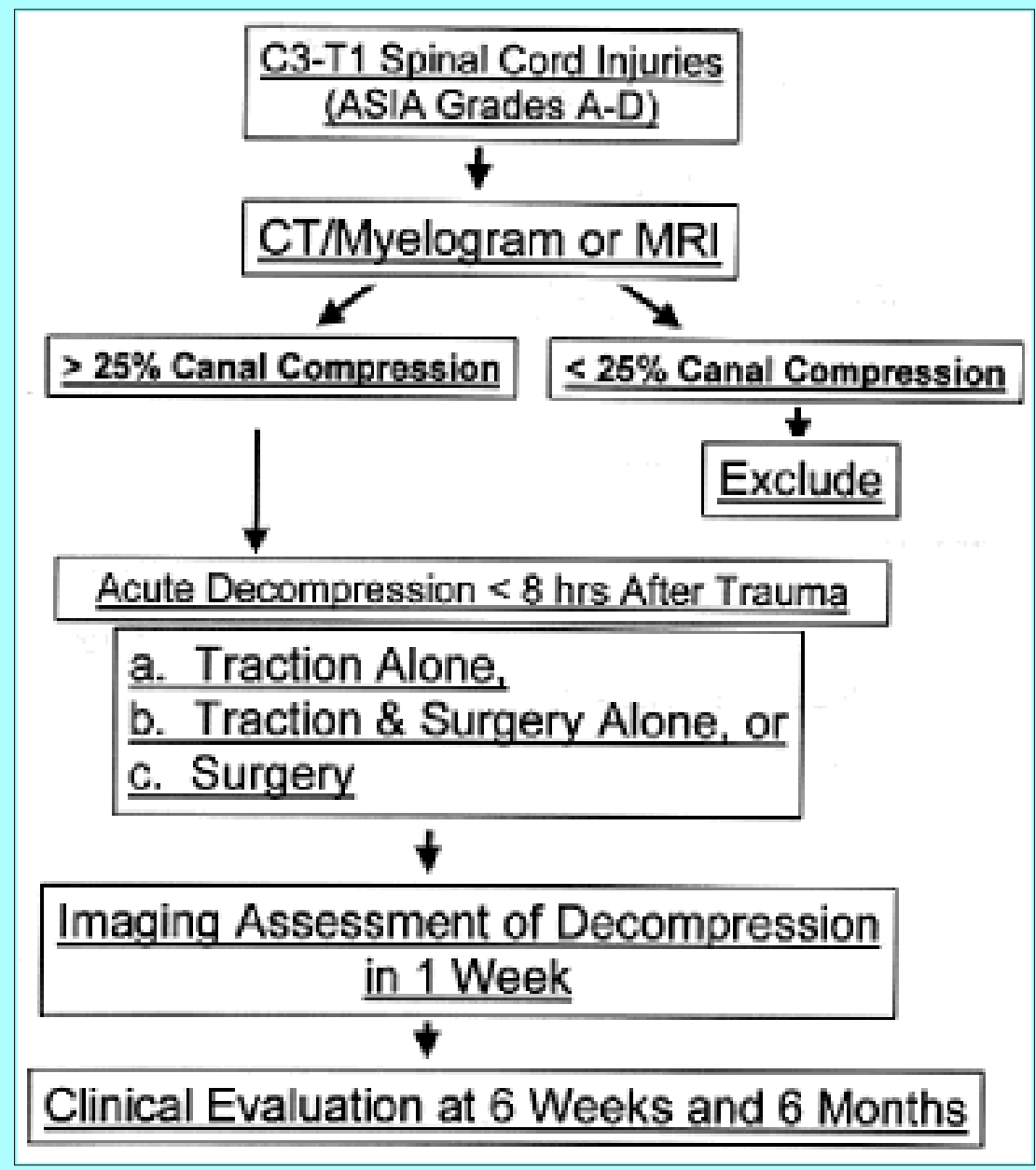

Fig. 1. Flow chart showing the pilot study design. The protocol required immediate MR imaging or CT/myelography followed by acute decompressive procedures by 8 hours of injury in those patients with subaxial cervical spine injuries (C3-T1). One of the three methods of decompression was used: 1) traction alone; 2) traction and surgery; or 3) surgery alone.

\section{RESULTS}

A total of 26 patients were accrued from the eight North American centers from October 1, 1996, to January 31,1997 . It was estimated that this number represented less than $10 \%$ of patients with cervical spinal cord injuries admitted to these centers during this period. There were 22 men $(84.6 \%)$ and four women $(15.2 \%)$, with an average age of $30.3 \pm 2.8$ years (standard error of the mean) and a range of 18 to 68 years. Seventy three percent of the patients were less than age 35 years. The main causes of spinal cord injury were traffic accidents (58\%), falls (15\%), sports-related activities (6\%), and violent events (4\%). At clinical examination on admission, 13 patients (50\%) were ASIA Grade A. All patients received postinjury intravenous methylprednisolone, but three patients $(11.5 \%)$ did not receive the dosage and/or regimen recommended in NASCIS-2.[6]

Significant difficulties were encountered in obtaining immediate imaging studies after acute SCI (Table 1). An average of $3.7 \pm 0.9$ hours (nine patients) was required for obtaining a CT/myelogram; and nearly 8 hours ( $7.5 \pm 1.2$ hours (21patients) elapsed before the MR imaging was performed. Six patients underwent both CT/myelography and MR imaging after admission. Fracture dislocation was the major 
radiological finding in 20 patients (76.9\%); and disc herniation was detected in 10 patients $(38.5 \%)$.

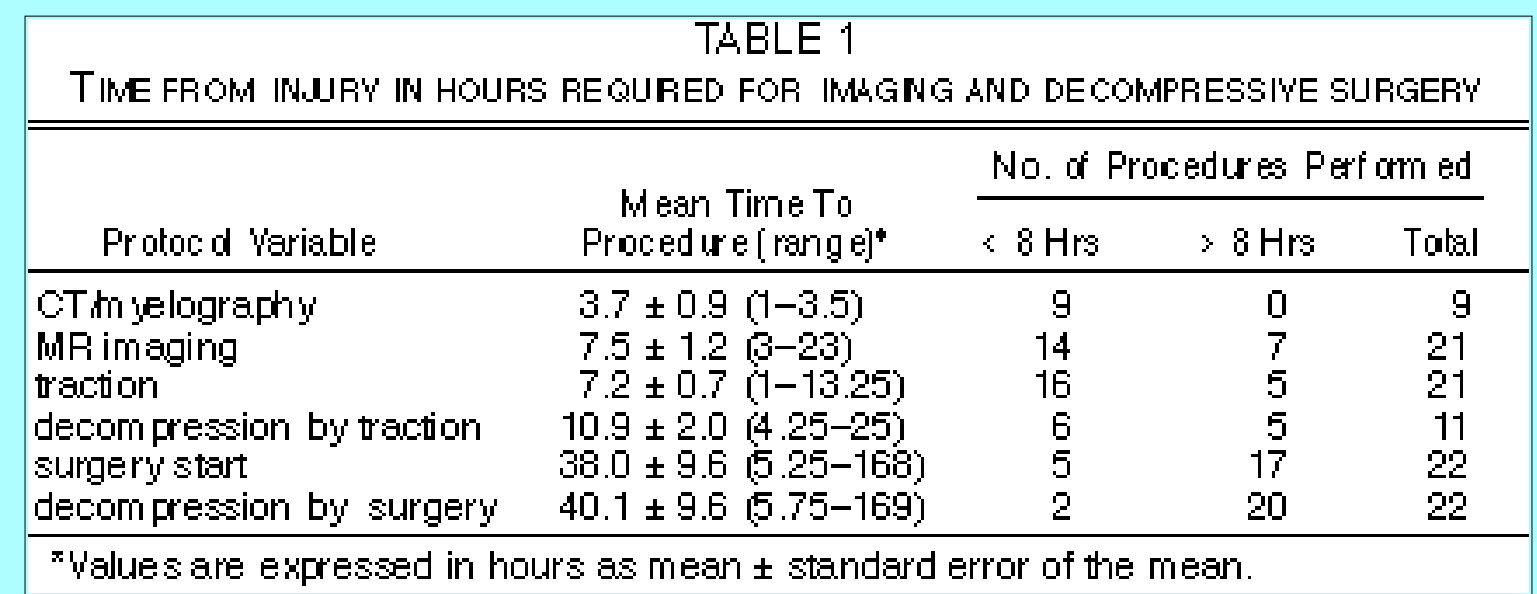

Traction combined with surgery was used as the method of decompression in most patients (17 patients, $65.3 \%)$. Traction alone was applied in four patients $(15.4 \%)$, and surgery alone was performed in five patients (19.2\%) (Fig. 2). An average $7.2 \pm 0.7$ hours postinjury was required for the application of traction in the 21 patients (80.8\%) (Table 1). Decompression by traction was achieved in only 11 patients $(42.3 \%)$ after an elapsed time of $10.9 \pm 2.0$ hours postinjury. In these 11 patients, decompressive procedures were completed in only six patients (23\%) within 8 hours of injury. Twenty-two patients (84.6\%) underwent surgical decompressive procedures $38.0 \pm 9.6$ hours postinjury. This surgical group consisted of seven patients who underwent decompressive traction, 10 for whom traction was unsuccessful, and five patients who did not undergo traction treatment. Of the 22 operations performed, 14 operations were from an anterior cervical approach, six a posterior approach, and two operations required a combined approach. Another 2 hours were required before decompressive surgery was achieved (40.1 \pm 9.6 hours; 22 patients) (Table 1). Only two patients $(7.6 \%)$ underwent a surgical decompressive procedure within 8 hours postinjury. The surgical decompressive procedure was achieved in seven patients $(27.0 \%)$ within 12 hours of injury. 


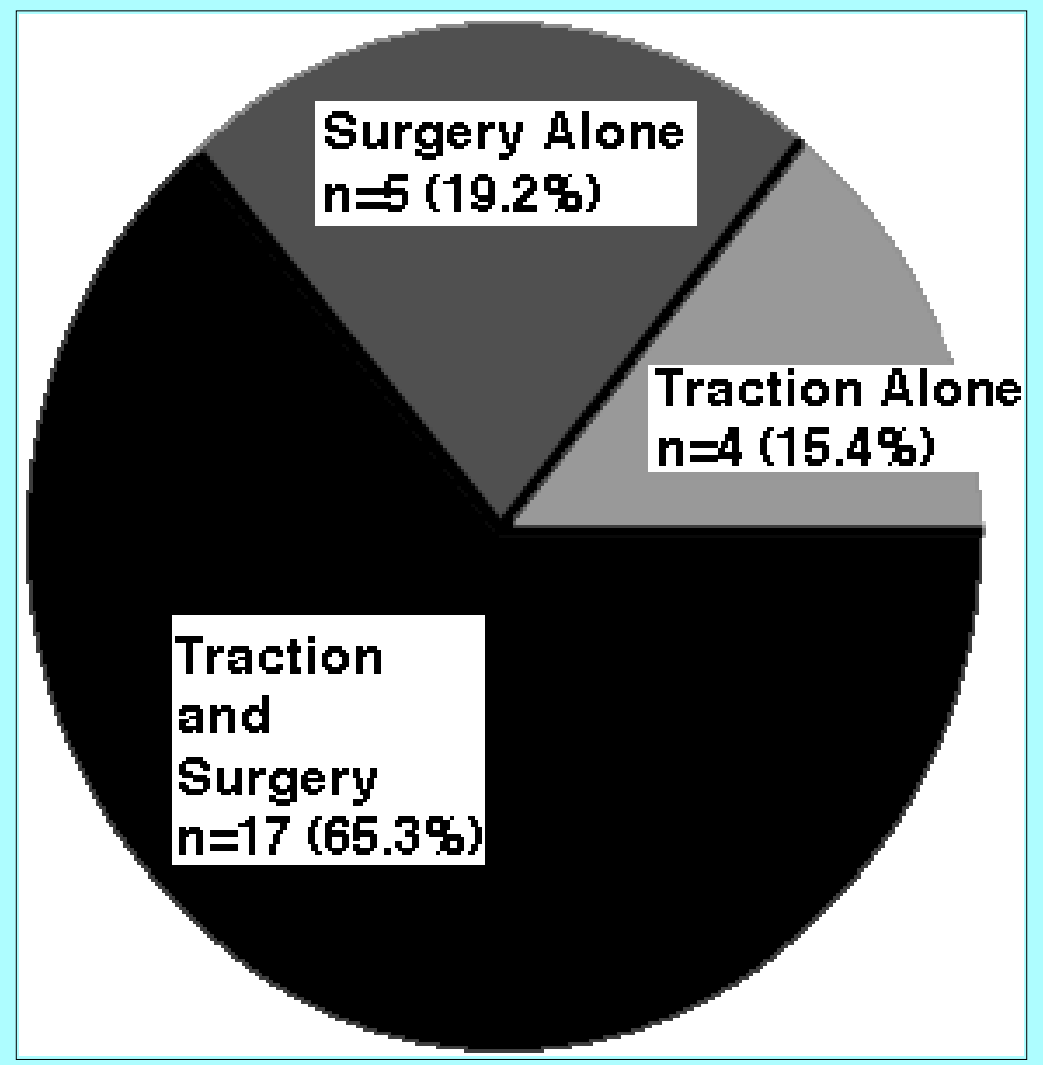

Fig. 2. Pie chart showing the methods of decompression and the number and percentage of patients who underwent each decompressive procedure.

Neurological outcome was assessed at 6 weeks and 6 months from the time of injury using the ASIA[3] and NASCIS systems[5,6] to score neurological status and motor and sensory recovery (Table 2). One patient died of overwhelming sepsis and pneumonia 2 months postinjury. Of the 13 patients who presented with complete injury of ASIA Grade A, 11 (84.6\%) remained Grade A at 6 months. Five of 26 (19.2\%) patients improved from Grade D to E at 6 months, recovering normal motor and sensory function, whereas there were no Grade E patients at the time of admission. These five patients underwent decompressive treatment at an average time of $30.8 \pm 14.1$ hours postinjury. Traction followed by surgery was the decompressive method in four patients in this group and traction alone was applied in one patient.

\begin{tabular}{|ccccccc|}
\hline \multicolumn{7}{c|}{ TABLE 2 } \\
\multicolumn{7}{|c|}{ ASIA GRADE IN 26 PATIENTS WITH ACUTE SCI ON ADMISION } \\
AND AT 6-MON THS \\
\hline \hline $\begin{array}{c}\text { ASA } \\
\text { Grade on } \\
\text { Admission }\end{array}$ & A & B & C & D & E & Total \\
\hline A A & 11 & & 1 & 1 & & 13 \\
B & & 1 & 1 & 1 & 1 & 4 \\
C & & & & 2 & & 2 \\
D & & & & 2 & 5 & 7 \\
E & & & & & & 0 \\
\hline
\end{tabular}

\section{DISCUSSION}

Recent advances in the safety and efficacy of surgical decompressive techniques to treat the spinal cord 
injuries offer significant potential for repairing some of the damage caused by spinal injuries.[21,25] Furthermore, surgical decompressive procedures are commonly performed in North America to treat patients with acute SCI. For example, Tator, et al.[21] have observed that $56 \%$ of a prospective series of 208 patients with acute SCI underwent at least one spinal operation, and in a retrospective series of 1385 patients, $75 \%$ of patients underwent surgical treatment.[2] However, controversy exists as to the ideal timing (early versus late) and the benefits derived from undergoing acute decompressive seurgery after acute SCI.[16,23] Thus, the role of acute decompressive surgery in improving neurological recovery remains unclear.

Proponents for early surgical decompressive procedures argue that early intervention may halt the rapid progression of pathological changes known to occur in the injured spinal cord and, thus, may also prevent neurological deterioration in the patients.[5,6,22] Early surgery may allow an earlier start of the rehabilitation process, thus decreasing the overall expense of managing patients with SCIs.[23] On the other hand, authors of several reports in the literature warn against performing early surgery in these critically injured and medically unstable patients.[17, 26] Marshall, et al.[17] found that more patients worsened neurologically after early surgery $(<5$ days). However, modern methods of resuscitation and life support have allowed these patients to undergo surgery with minimal differences in complication rates between operative and nonoperative cases.[19,21,25]

Results from the recent NASCIS-2[5] and NASCIS-3[6] trials have provided important insights into the benefits of early intervention in patients with SCIs. The use of acute pharmacotherapy with methylprednisolone can attenuate the secondary injury cascade and enhance neurological recovery if administered within 8 hours of acute SCI. In contrast, patients treated with methylprednisolone more than 8 hours postinjury showed no significant difference in neurological recovery compared with patients who received a placebo.[5] Experimental and clinical evidence is accumulating to suggest that acute decompressive surgery performed in the injured spinal cord within a few hours of injury may also result in improved neurological recovery. Several experimental studies of decompressive surgery in the injured spinal cord have been performed in laboratory animals; most found that neurological recovery is enhanced by early decompressive surgery performed to treat the acutely compressed spinal cord in a variety of animal species injured by a variety of models of acute compression. $[7,9,13]$ Because persistent compression of the spinal cord is a potentially reversible form of secondary injury, the duration of spinal compression was an important factor affecting neurological recovery in these animals. $[9,13]$ There have also been many clinical studies, almost all retrospective, that were performed to determine whether early decompressive surgery improves neurological recovery in humans with acute SCI.[1,2,4,10,11,14,16,18,21,24,27] In most of these studies, the early surgery group underwent surgery 24 hours or more after injury, and in general, there was no relationship between the timing of surgery and neurological recovery. Furthermore, in a recent randomized controlled study by Vaccaro, et al.[23] it was revealed that no significant neurological benefit with early surgery $(<72$ hours) was achieved when compared with late surgery ( $>5$ days). However, the results of two encouraging studies have demonstrated enhanced in neurological recovery when traction reduction procedures are applied in cervical SCI cases in less than 8 hours.[1,8] To date, no randomized controlled trial has been undertaken to examine whether acute decompressive procedures performed within this critical 8-hour time window can improve neurological outcome. Thus, the present pilot study was designed to determine the feasibility of performing acute decompressive procedures within 8 hours of injury as a preparatory step to examining whether this treatment of the injured cervical spinal cord within this time frame can improve outcome in a prospective randomized controlled trial. 
This STASCIS pilot study showed that the 8-hour interval from trauma to decompressive procedure was too short to permit enrollment of large numbers of patients into the prospective study. Significant difficulties were encountered in many centers in obtaining MR imaging studies in patients with acute SCI (Table 1). The delay in treatment may also have been related to the tertiary referral patterns present in the participating centers. The combination of required time for rescue, resuscitation, transport, imaging, and surgical preparation usually exceeded the 8-hour injury-to-decompression window. Only a small number of patients underwent initial baseline MR imaging and decompressive procedure within the 8-hour time interval. It is believed that earlier transfer of patients to the acute injury center can be accomplished through educational efforts directed toward first aid personnel and emergency physicians. Additionally, earlier access to MR imaging should be made possible by enlisting the cooperation of the radiologists. This is of particular importance, as indicated by the recent study from our STASCIS group, which found that CT scanning alone was insufficiently accurate to predict which patients with acute SCI had spinal cord compression.[12]

That most of the patients with complete neurological injury (ASIA Grade A) did not improve confirms the irreversible and devastating nature of SCI. However, neurological recovery to normal motor and sensory function (ASIA Grade E) was observed in some patients with Grade D. Because this pilot study involved only a small number of patients and was not designed to determine the neurological benefits of early surgery, the significance of the observed improvement is not known. This issue can only be resolved by studying a large number of patients in a prospective randomized controlled trial.

In summary, we showed that, under the system of care in place at the study centers, it was not feasible to establish a radiological diagnosis of spinal cord compression and to perform decompressive procedures within 8 hours of injury. Indeed, only seven (26.9\%) of the 26 patients underwent decompressive surgery within 12 hours. The information gained from this study has allowed us to identify the principal factors that delay early decompressive surgery, the most important of which were delayed referral and admission to the centers and delayed imaging studies. It is believed that these factors can be remedied to allow the development of a protocol for decompressive procedures within 8 hours of injury.

\section{Appendix}

Members of the participating STASCIS Surgical Decompression Study Group.

Brian Cuddy, Medical University of South Carolina, Charleston, South Carolina

Curtis Dickman, Volker Sonntag, Barrow Neurological Institute, Phoenix, Arizona

Mahmood Fazl, Sunnybrook Health Sciences Center, Toronto, Ontario, Canada

Michael Fehlings, Charles Tator, The Toronto Hospital, Toronto, Ontario, Canada

Barth Green, University of Miami, Miami, Florida

Patrick Hitchon, University of Iowa Hospital and Clinics, Iowa City, Iowa

Bruce Northrup, Thomas Jefferson University, Philadelphia, Pennsylvania

Frank Wagner, University of California, Davis Medical Center, Sacramento, California 


\section{References}

1. Aebi M, Mohler J, Zach GA, et al: Indication, surgical technique, and results of 100 surgically-treated fractures and fracture-dislocation of the cervical spine. Clin Orthop 203:244-257, 1986

2. Ahn JH, Ragnarsson KT, Gordon WA, et al: Current trends in stabilizing high thoracic and thoracolumbar spinal fractures. Arch Phys Med Rehab 65:366-369, 1984

3. American Spinal Injury Association: Standards for Neurological and Functional Classification of Spinal Cord Injury. Revised Edition. Chicago, Ill: American Spinal Injury Association, 1992

4. Benzel EC, Larson SJ: Functional recovery after decompressive spine operation for cervical spine fractures. Neurosurgery 20:742-746, 1987

5. Bracken MB, Shepard MJ, Collins WF, et al: A randomized controlled trial of methylprednisolone or naloxone in the treatment of acute spinal cord injury. Results of the Second National Acute Spinal Cord Injury Study. N Engl J Med 322:1405-1411, 1990

6. Bracken MB, Shepard MJ, Holford TR, et al: Administration of methylprednisolone for 24 or 48 hours or tirilazad mesylate for 48 hours in the treatment of acute spinal cord injury. Results of the Third National Acute Spinal Cord Injury Randomized Controlled Trial. JAMA 277:1597-1604, 1997

7. Brodkey JS, Richards DE, Blasingame JP, et al: Reversible spinal cord trauma in cats. Additive effects of direct pressure and ischemia. J Neurosurg 37:591-593, 1972

8. Cotler JM, Herbison GJ, Nasyuti JF, et al: Closed reduction of traumatic cervical spine dislocation using traction weights up to 140 pounds. Spine 18:386-390, 1993

9. Dolan EJ, Tator CH, Endrenyi L: The value of decompression for acute experimental spinal cord compression injury. J Neurosurg 53:749-755, 1980

10. Donovan WH, Kopaniky D, Stolzmann E, et al: The neurological and skeletal outcome in patients with closed cervical spinal cord injury. J Neurosurg 66:690-694, 1987

11. Duh M, Shepard MJ, Wilberger JE, et al: The effectiveness of surgery on the treatment of acute spinal cord injury and its relation to pharmacological treatment. Neurosurgery 35:240-249, 1994

12. Fehlings MG, Rao S, Tator $\mathrm{C}$, et al: The optimal radiological method to assess spinal canal compromise and cord compression in patients with cervical spinal cord injury: Part II: results of a multi-center study. Spine (In press, 1999)

13. Guha A, Tator $\mathrm{CH}$, Endrenyi L, et al: Decompression of the spinal cord improves recovery after acute experimental spinal cord compression injury. Paraplegia 25:324-339, 1987

14. Hadley MN, Fitzpatrick BC, Sonntag VK, et al: Facet fracture-dislocation injuries of the cervical spine. Neurosurgery 30:661-666, 1992

15. Kraus JF, Franti CE, Riggins RS, et al: Incidence of traumatic spinal cord lesions. J Chron Dis 28:471-492, 1975

16. Levi L, Wolf A, Rigamonti D, et al: Anterior decompression in cervical spine trauma: does the timing of surgery affect the outcome? Neurosurgery 29:216-222, 1991 
17. Marshall LF, Knowlton S, Garfin SR, et al: Deterioration following spinal cord injury. A multicenter study. J Neurosurg 66:400-404, 1987

18. Maynard F, Reynolds GG, Fountain S, et al: Neurological prognosis after traumatic quadriplegia.

Three year experience of California Regional Spinal Cord Injury Care System. J Neurosurg 50:611-626, 1979

19. Rizzolo SJ, Vaccaro AR, Cotler JM: Cervical spine trauma. Spine 19:2288-2298, 1994

20. Stripling TE: The cost of economic consequences of traumatic spinal cord injury. Paraplegia News:50-54, August, 1990

21. Tator CH, Duncan EG, Edmonds VE, et al: Comparison of surgical and conservative management in 208 patients with acute spinal cord injury. Can J Neurol Sci 14:60-69, 1987

22. Tator $\mathrm{CH}$, Fehlings MG: Review of the secondary injury theory of spinal cord trauma with emphasis on vascular mechanisms. J Neurosurg 75:15-26, 1991

23. Vaccaro AR, Daugherty RJ, Sheehan TP, et al: Neurologic outcome of early versus late surgery for cervical spinal cord injury. Spine 22:2609-2613, 1997

24. Waters RL, Adkins RH, Yakura JS, et al: Effect of surgery on motor recovery following traumatic spinal cord injury. Spinal Cord 34:188-192, 1996

25. Wilberger J: Diagnosis and management of spinal cord trauma. J Neurotrauma 8 (Suppl 1):S21-S30, 1991

26. Wilmot CB, Hall KM: Evaluation of the acute management of tetraplegia: conservative versus surgical treatment. Paraplegia 24:148-153, 1986

27. Wolf A, Levi L, Mirvis S, et al: Operative management of bilateral facet dislocation. J Neurosurg 75:883-890, 1991

Manuscript received December 12, 1998.

Accepted in final form December 18, 1998.

Address reprint requests to: C. H. Tator, M.D., Division of Neurosurgery, Toronto Western Hospital, 399 Bathurst Street, Toronto, Ontario, M5T 2S8, Canada. 